\title{
Factores que afectan la vida útil de vacas doble propósito
}

\author{
Factor affecting the useful life of dual-purpose cows
}

\author{
Leidys Murcia R, ${ }^{1}$ Ing Agr, Gonzalo Martínez G, ${ }^{1 *}$ Ph.D.
}

${ }^{1}$ Universidad Central de Venezuela. Instituto de Producción Animal, Facultad de Agronomía. Apartado Postal 4579. Maracay, estado Aragua. Venezuela. Correspondencia: gemg7235@gmail.com

Recibido: Junio de 2011; Aceptado: Agosto de 2012.

\section{RESUMEN}

Objetivo. Determinar el efecto de factores no genéticos y de grupo racial, sobre la vida útil (VU) de vacas doble propósito. Materiales y métodos. Se utilizó información de 2098 vacas, que incluyó a todas las hembras que ingresaron a servicio y salieron del sistema productivo. La VU se cuantificó como el total de partos al salir del rebaño. Los efectos incluidos en el modelo fueron hacienda (H: AG, VV), año de primer parto (A1P: 1995-2006), época de primer parto (E1P: Enero-marzo, abril-mayo, junio-agosto, septiembre-diciembre) grupo racial (GR: C, 50C50E, ML) y las interacciones HxGR y A1PxGR. Resultados. La media de VU fue de 2.63 partos \pm 0.07 . Todos los factores tuvieron efectos altamente significativos $(p<0.01)$ sobre $\mathrm{VU}$, menos $\mathrm{H}$. Es interesante resaltar que las interacciones $\mathrm{HxGR}$ y A1PxGR fueron altamente significativas $(p<0.01)$ indicando que los grupos raciales se comportan diferente en cada hacienda y en el transcurso de los años. Conclusiones. Los efectos no genéticos y de grupo racial tienen una alta influencia sobre VU.

Palabras clave: Longevidad, razas de doble propósito, rendimiento lechero, reproducción (Fuente:CAB).

\section{ABSTRACT}

Objective. Determine the effect of non-genetic factors and breed group, on the useful life (UL) of dual-purpose cows. Materials and methods. Records from 2098 cows were used in the analysis which included all the females who entered to the reproductive program and were culled or died. The data came from two dual-purpose commercial herds located in the basin of the Lake of Maracaibo in Trujillo State. The UL was quantified in number of calving upon culling. The parameters included in the model were: herd (H: AG, VV), year of the first calving (Y1C: 1995-2006), season of the first calving (S1C: January-March, April-May, June-August, September-December), breed group (BG: Zebu, 50\%Zebu50European; ZE, Milking Crossbred; MC) and the interaction HxBG y Y1CxBG. Results. The UL average was $2.63(0.07)$ calvings. All the factors had highly significant effects $(p<0.01)$ on UL, however, $\mathrm{H}$ did not affect UL. It is interesting to highlight that HXBG and Y1CxBG interactions were highly significant $(p<0.01)$ indicating that breed groups behave differently in every herd and during the course of the years. Conclusions. Non-genetic factors and breed group have a high influence on UL.

Key words: Longevity, dual purpose breeds, milk production, reproduction (Source:CAB). 


\section{INTRODUCCIÓN}

La ganadería bovina doble propósito es un sistema de producción de leche y carne, que ha surgido como una respuesta a las condiciones socioeconómicas y ambientales de los productores venezolanos y de América Latina tropical, permitiendo producir leche y carne en un sólo sistema productivo, con la finalidad de hacer un uso sostenible, eficiente y rentable de los recursos naturales, animales, humanos y económicos que se encuentren disponibles, para satisfacer las necesidades alimentarías de la población y generar ingresos al productor (1).

Los caracteres que influencian la eficiencia productiva de los sistemas de doble propósito se dividen en: productivos (leche y carne), y funcionales o secundarios (fertilidad, longevidad, entre otros) (2). Los caracteres funcionales son considerados como los que aumentan la eficiencia económica mediante la reducción de los gastos e inversiones, lo que conlleva a un aumento en la cantidad de productos vendibles (3). Entre estos caracteres destaca la longevidad, la cual, expresa el tiempo (días, meses, años) que un animal permanece en el rebaño, desde el primer parto hasta el descarte, es decir, su tiempo de vida productiva en el rebaño. Existen otras medidas que expresan mejor la longevidad de las vacas desde el punto de vista de utilidad y productividad como lo son la vida útil (VU), la cual expresa el número de eventos productivos en la vida del animal (número de partos, número de lactancias, entre otros) y vida productiva (VP) expresada como un valor total que representa la producción en la vida de un animal (producción de leche acumulada de por vida, peso total de becerros nacidos y destetados, entre otros) $(2,4)$.

La longevidad de las vacas es una característica compleja que refleja su desempeño en la vida total y está determinada principalmente por la sobrevivencia, salud, fertilidad, habilidad materna, así como su capacidad de adaptación al ambiente, es decir, la virtud que tiene un animal para agradar a su propietario, tanto por sus características productivas o fenotípicas como por no ser problemático (5).

A pesar de que en Venezuela, la ganadería doble propósito ha sido uno de los sistemas que se adapta a diversas condiciones agroecológicas, presentándose zonas favorables para el óptimo desarrollo de la ganadería y también ambientes limitantes que afecta. La producción de las vacas y manejo de los rebaños, se puede decir que tanto la producción como la reproducción en estos sistemas se ve afectada por un sin fin de factores ambientales, así como el manejo y la disponibilidad de recursos. Entre estos factores el efecto finca es en general la fuente de variación más importante con diferencias extremas de 1,7 partos, para fincas ubicadas en una misma localidad (6-8). El año del primer parto es otro de los factores que más influye sobre la VU, debido a las variaciones de las condiciones temporales, de manejo o la política de selección que varían de un año a otro, la literatura citada reporta una diferencia de hasta 2 partos entre años extremos $(6,7,9)$.

El comportamiento productivo de vacas ubicadas en una misma finca, está influenciado por factores genéticos, (principalmente diferencias entre grupo raciales) siendo las hembras con $50 \%$ herencia europea las que presentan los mejores promedios de vida útil y productiva, los que peor se comportan son los de menor herencia europea, encontrando diferencias extremas hasta de 1.2 partos entre los grupos raciales con $50 \%$ herencia europea y los menor a $50 \%$ europea, este comportamiento puede variar dependiendo del manejo que se tenga en cada finca $(3,5,8)$. En vista de que existe poca información sobre la VU de vacas doble propósito se planteó como objetivo de la presente investigación determinar el efecto de los factores no genéticos como: hacienda, año, época de primer parto y grupo racial, sobre la vida útil de vacas de doble propósito.

\section{MATERIALES Y MÉTODOS}

Sitio de estudio. El estudio se realizó con datos provenientes de dos unidades de producción comerciales los cuales corresponden a la hacienda Valle Verde (VV) ubicada en el Municipio La Ceiba, Parroquia Santa Apolonia, Estado Trujillo y a la Agropecuaria Agrounica (AG), localizada al norte del estado Trujillo limitando con el estado Zulia en el Municipio Andrés Bello, Parroquia Santa Isabel. Las cuales se encuentran situadas geográficamente al Sur del lago de Maracaibo, en el sector Trujillano de la Depresión de Lago de Maracaibo, caracterizado por un clima cálido y húmedo, con temperaturas promedio anuales superiores a los $28^{\circ} \mathrm{C}$ y precipitaciones promedio anuales entre 900 y $1300 \mathrm{~mm}$. Durante el año la distribución de las lluvias está bien definida, existiendo un pico de mayor precipitación entre los meses de septiembre a diciembre y otro pico de precipitación menos pronunciado entre abril y mayo. De acuerdo con el clima la zona de vida corresponde al Bosque húmedo tropical (Bht) (10).

Descripción general. El manejo de ambas fincas fue similar, teniendo como actividad principal la producción de leche. Los grupos raciales predominantes en los datos utilizados 
estuvieron conformados por animales como se describe a continuación: mayormente Cebú (>75\% herencia Bos indicus), 50\% Bos taurus 50\% Bos indicus y mestizos lecheros que son animales cuya composición racial exacta no puede ser determinada pero es típico de los rebaños de doble propósito.

El ordeño se realizó en dos turnos, el primero entre las 02:00 y 03:00 horas y el segundo a partir de las 14:00 horas, de forma manual en AG y mecánico en VV. En ambas haciendas se usó la modalidad de apoyo del becerro, seguido por el amamantamiento restringido por 30 minutos. La producción de leche se registró semanalmente.

En VV todas las hembras están en un programa de inseminación artificial. Las novillas son servidas por primera vez en promedio a los 33 meses con un peso entre $320-340 \mathrm{~kg}$. En AG se emplea el programa de inseminación artificial en una parte del rebaño (novillas y vacas secas), el peso promedio para el primer servicio es de 330 a $350 \mathrm{~kg}$ a una edad promedio de 29 meses, el resto de las vacas se encuentra bajo monta natural. En ambas haciendas para la detección de celo se utiliza la ayuda de un toro retajo o vaca androgenizada. Igualmente, en ambas haciendas las vacas que no quedan preñadas a partir de tres inseminaciones pasan seis meses en monta natural, de no ser positivas en la próxima palpación son eliminadas.

La fuente principal de la alimentación la constituyen los pastos, dentro de los cuales destaca la presencia de tanner (Urochloa radicans), guinea (Panicum maximun), y estrella (Cynodon plectostachyus y Cynodon nlemfuensis) para $\mathrm{VV}$, mientras que en $\mathrm{AG}$ además de las anteriores se encuentran las especies: pará (Brachiaria mutica), y alemán (Echynochloa polystachya). Todo el rebaño se suplementa con minerales y sal ad libitum, salvo los becerros pre-destete que reciben aproximadamente 0.5 $\mathrm{kg} /$ día de un alimento balanceado comercial, aunque esta práctica no es rutina de manejo, ya que existen cambios en cada finca cada año.

El plan sanitario es el mismo en ambas explotaciones, siguiendo un cronograma de actividades durante todo el año, según el grupo etario y de la época del año, siguiendo las recomendaciones oficiales y del médico veterinario que orienta cada hacienda. Anualmente se hacen pruebas serológicas para el diagnóstico de brucelosis y tuberculosis, y se vacuna anualmente contra fiebre aftosa, enfermedades clostridiales y brucelosis (todas las hembras entre tres y ocho meses), además se realizan desparasitaciones internas y externas trimestralmente.
Análisis estadístico. Para el análisis se tomaron sólo aquellas vacas que ingresaron a servicio y salieron del sistema productivo ya sea por muerte o descarte, distribuidas desde el año de primer parto 1995 al 2006 como se observa en la tabla 1, y la base de datos quedó conformada por 2098 observaciones, constituidas por vacas cebú y de diferentes grados de mestizaje Bos taurus x Bos indicus.

Tabla 1. Distribución del número de observaciones según finca y año de primer parto.

\begin{tabular}{llll}
\hline & \multicolumn{2}{c}{ Fincas } & Total \\
\cline { 2 - 4 } Años & AG & VV & \\
\hline 1995 & 119 & 47 & 166 \\
1996 & 89 & 24 & 113 \\
1997 & 112 & 53 & 165 \\
1998 & 197 & 41 & 238 \\
1999 & 163 & 91 & 254 \\
2000 & 169 & 69 & 238 \\
2001 & 182 & 43 & 225 \\
2002 & 131 & 58 & 189 \\
2003 & 135 & 33 & 168 \\
2004 & 89 & 47 & 106 \\
2005 & 84 & 22 & 196 \\
\hline Total & 1551 & 547 & \\
\hline
\end{tabular}

La variable en estudio fue vida útil (VU) medida como el número total de partos al salir el rebaño (muerte o descarte) para todas las hembras que para el año 2006 tuvieron al menos tres oportunidades de parto utilizando la definición de grupo de oportunidad de Hudson y Van Vleck (11).

Para el procesamiento de los datos se realizó un análisis de varianza a través de un modelo lineal de efectos fijos, por el método de máxima verosimilitud restringida utilizando PROC MIXED de Statistical Analysis System versión 9.1 (12) que permite analizar efectos con desigual número de observaciones.

Los efectos incluidos fueron: Hacienda (H:AG,VV), año de primer parto (A1P: 1995-2006), época de primer parto $(E 1 P$ :E1=enero-marzo, E2=abrilmayo, E3=junio-agosto, E4=septiembrediciembre) y grupo racial (GR: $C=\geq 75 \%$ herencia Bos indicus, $\mathrm{CE}=50 \%$ Bos taurus $50 \%$ Bos indicus y $\mathrm{ML}=$ mestizos lecheros). 
El modelo estadístico utilizado fue: $Y_{i j k l m}=\underset{ }{(A 1 P x G R)_{j l}}+E_{i j k l m}^{\mu}+H_{i}+A 1 P_{k}+G P_{l}+(H \times G R)_{i l}+$

$Y_{i j k l m}=$ Vida útil de un animal "m" de la hacienda "i" parida en el año "j", en la época "k", del grupo racial "I".

$\mu=$ Media teórica de VU.

$\mathrm{H}_{\mathrm{i}}=$ Efecto de hacienda ( $\left.\mathrm{i}=\mathrm{AG}, \mathrm{VV}\right)$.

$A 1 P_{j}=$ Efecto de año de primer parto $(j=1995, \ldots$, 2006).

$\mathrm{E}_{1} \mathrm{P}_{\mathrm{k}}=$ Efecto de la época de primer parto $(\mathrm{k}=$ $E 1, \ldots E 4)$.

$\mathrm{GR}_{1}=$ Efecto del grupo racial de la vaca ( $\mathrm{I}=\mathrm{C}$, $C E, M L)$.

$(\mathrm{H} \times \mathrm{GR})_{\mathrm{il}}=$ Efecto de la interacción hacienda $\mathrm{x}$ grupo racial.

$(A 1 P \times G R)_{j 1}=$ Efecto de la interacción año de primer parto $x$ grupo racial.

$\mathrm{E}_{\text {ijklmn }}=$ Efecto del error experimental, normal e independientemente distribuido con media cero y varianza $\sigma^{2}$.

Las interacciones no presentadas en el modelo previamente descrito fueron descartadas ya que en el pre-análisis no resultaron significativas a un nivel mínimo de $p<0.05$. Las diferencias entre medias fueron establecidas utilizando la prueba de t de "Student" (13).

Las épocas de nacimiento (seca o lluviosa) fueron definidas de acuerdo con los períodos de crecimientos utilizando la información de precipitación y evapotranspiración de la finca o de la estación climatológica más cercana. La época seca fue definida como aquellos meses donde la evapotranspiración supera a la precipitación y por ende viceversa para los meses lluviosos, quedando conformada de la siguiente forma: época 1 (E1: enero a marzo), época 2 (E2: abril y mayo), época 3(E3: junio a agosto) y época 4 (E4: septiembre a diciembre).

\section{RESULTADOS}

Promedio de VU y análisis de varianza. Se detectaron diferencias altamente significativas $(p<0.01)$, para los factores A1P, E1P, GR, HxGR y $A 1 P x G R$, siendo $H$ el único que no mostró diferencias estadísticas $(p>0.05)$. Los factores que ejercieron mayor influencia sobre la VU en orden de importancia son: A1P, HxGR y GR.

La VU promedio fue de 2.63 partos, con valores extremos de 4.7 y 1 parto, tal como se puede observar en la tabla 2.

\section{Factores no genéticos y de grupo racial que afectan la vida útil.}

Efecto Hacienda. Este factor no mostró efecto estadísticamente significativo $(p=0.192)$ sobre la VU (Tabla 2). En general, el menor promedio de VU se obtuvo para la hacienda AG con 2.58 partos, mientras que $\mathrm{VV}$ presentó un promedio de 2.7 partos, con una diferencia de 0.12 partos (ee $=0.095 ; p=0.192$ ) entre ambas fincas.

Efecto año de primer parto. El efecto año de primer parto sobre la vida útil de las vacas resultó altamente significativo $(p<0.01)$, con una tendencia decreciente de vida útil a lo largo de los años en estudio, tal como se observa en la tabla 3 , estos resultados concuerdan con los obtenidos por Ponce de León y Guzmán (8) y Florio (9).

Efecto de la época de primer parto sobre la VU. Se encontraron diferencias significativas en VU $(p=0.017)$ con respecto a la E1P de la vaca. Las medias ( $n$, ee) por E1P fueron las siguientes: $2.78(570,0.08), 2.68(352,0.09)$, $2.59(502,0.08)$ y $2.50(674,0.07)$ para E1, E2, E3 y E4, respectivamente. Existió una diferencia entre las épocas extremas (E1 y E4) de 0.28 partos (ee $=0.09$ partos; $p<0.002$ ) la cual ocurre entre las épocas de enero-marzo y septiembrediciembre

Tabla 2. Efecto de hacienda, grupo racial y de la interacción hacienda x grupo racial sobre la vida útil en vacas doble propósito.

\begin{tabular}{|c|c|c|c|c|c|c|c|c|c|}
\hline \multirow{3}{*}{ Grupo Racial } & \multicolumn{6}{|c|}{ Hacienda } & \multirow{2}{*}{\multicolumn{3}{|c|}{ Total }} \\
\hline & \multicolumn{3}{|c|}{ AG } & \multicolumn{3}{|c|}{$\mathbf{v v}$} & & & \\
\hline & $\mathbf{n}$ & M & ee & $\mathbf{n}$ & M & ee & $\mathbf{N}$ & M & Et \\
\hline $\mathrm{C}$ & 179 & $2.66^{b}$ & 0.14 & 86 & $2.73^{a b}$ & 0.18 & 265 & $2.69^{\mathrm{ab}}$ & 0.12 \\
\hline CE & 984 & $3.11^{\mathrm{a}}$ & 0.05 & 169 & $2.49^{b}$ & 0.13 & 1153 & $2.80^{\mathrm{a}}$ & 0.07 \\
\hline ML & 388 & $1.97^{c}$ & 0.10 & 292 & $2.88^{a}$ & 0.11 & 680 & $2.42^{\mathrm{b}}$ & 0.08 \\
\hline Total & 1551 & 2.58 & 0.06 & 547 & 2.70 & 0.08 & 2098 & 2.67 & 0.04 \\
\hline
\end{tabular}

AG: Agrounica; VV: Valle Verde; C: Acebuado; CE: 50\% Cebú - 50\% Europeo; ML: Mestizo Lechero, M: media, n: número de observaciones, ee: error estándar. Letras distintas tanto en filas como en columnas indican diferencias a $\mathrm{p}<0.05$. 
Tabla 3. Efecto del año del primer parto sobre la vida útil en vacas doble propósito.

\begin{tabular}{cccc}
\hline $\begin{array}{c}\text { Efecto } \\
\text { Año de primer parto }\end{array}$ & N & Media & ee \\
\hline 1995 & 166 & $4.70^{\mathrm{a}}$ & 0.14 \\
1996 & 113 & $4.57^{\mathrm{a}}$ & 0.17 \\
1997 & 165 & $3.93^{\mathrm{b}}$ & 0.15 \\
1998 & 238 & $2.96^{\mathrm{c}}$ & 0.13 \\
1999 & 254 & $2.89^{\mathrm{c}}$ & 0.11 \\
2000 & 238 & $2.46^{\mathrm{d}}$ & 0.12 \\
2001 & 225 & $2.52^{\mathrm{d}}$ & 0.14 \\
2002 & 189 & $1.95^{\mathrm{e}}$ & 0.16 \\
2003 & 168 & $1.85^{\mathrm{e}}$ & 0.17 \\
2004 & 136 & $1.46^{\mathrm{ef}}$ & 0.19 \\
2005 & 106 & $1.38^{\mathrm{ef}}$ & 0.26 \\
2006 & 100 & $1.01^{\mathrm{f}}$ & 0.24 \\
\hline
\end{tabular}

AG: Agrounica; VV: Valle Verde; CE: Acebuado; 50C50E: 50\% Cebú$50 \%$ Europeo; ML: Mestizo Lechero, $\mathrm{n}$ : número de observaciones. Letras distintas en filas indican diferencias a $(p<0.05)$.

Efecto del grupo racial sobre la vida útil. El GR afectó $(p=0.002)$ la variación de la VU. En la tabla 2 se puede observar como las vacas CE superan en vida útil a las vacas $C$ y las ML. No obstante, cabe destacar que al comparar las CE y las ML estas difieren en 0.37 partos (ee $=0.1$; $p<0.01)$. Tampoco se encontró diferencias $(p>0.05)$ entre vacas C y ML.

Efecto de la interacción $H \times$ GR sobre la VU. Esta interacción afectó la VU $(p<0.01)$, y los resultados se pueden apreciar en la tabla 2 . Existieron diferencias altamente significativas a favor de los animales $C E$, respecto a los $C$ y $M L$ en la hacienda $A G$, con una diferencia de 0.45 partos (ee $=0.15 ; p<0.002)$ y 1.14 partos (ee=0.10; $\mathrm{p}<0.0001)$, respectivamente, siendo las vacas ML las que obtuvieron el promedio más bajo de VU. Caso contrario a lo que sucede en la hacienda $\mathrm{VV}$ donde las vacas ML expresaron los mejores promedios de vida útil seguidas por las $\mathrm{C}$ y las CE fueron las de menor VU, con una diferencia a favor de las ML de 0.15 partos (ee $=0.21$; $p>0.4592)$ y 0.39 partos (ee $=0.17 ; p>0.05)$, con respecto a las $\mathrm{C}$ y $\mathrm{CE}$, respectivamente.

Se puede observar (Tabla 2) como las vacas ML de AG obtuvieron menor VU (0.92 partos; ee $=0.13 ; p<0.01$ ) que las $M L$ en $V V$, asimismo, las vacas CE de $A G$ superaron a las CE de $V V$ en 0.62 partos $(e e=0.14 ; p<0.01)$ y las vacas $C$ de $\mathrm{VV}$ fueron ligeramente inferiores en VU $(-0.07$ partos; ee $=0.22 ; \mathrm{p}>0.05$ ).

Efecto de la interacción A1P $x$ GR. La interacción $A 1 P \times G R$, tuvo un efecto significativo sobre la VU $(p<0.001)$. Como se observa en la figura 1 , hubo cambios tanto en la posición de los grupos raciales, como en la magnitud de las diferencias entre ellos a través de los años de estudio.

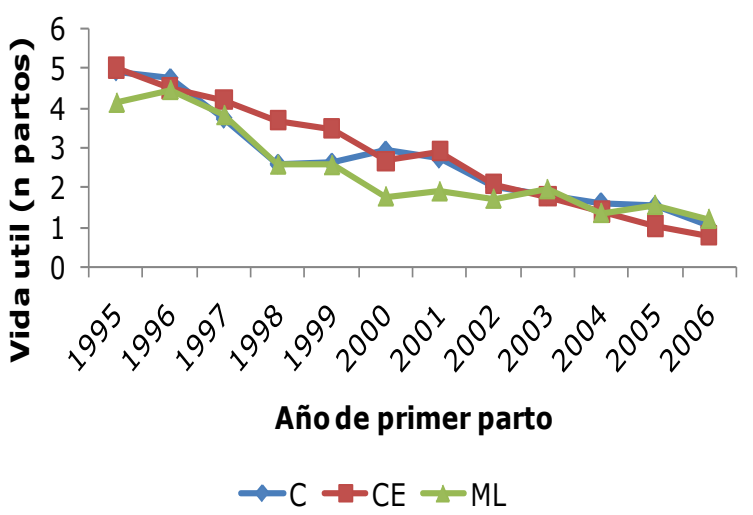

Figura 1. Efecto de la interacción grupo racial $x$ año de primer parto sobre la vida útil en vacas doble propósito.

En general, los animales ML tienen menor VU que vacas $C$ y CE. Sólo se encontró diferencias en VU entre las vaca C y CE para los años 1998 y 1999, con la mayor diferencia de 1.10 partos (ee $=0.36$; $p=0.0026)$ a favor de vacas CE. Las vacas CE superaron de forma significativa $(p<0.05)$ a ML en los años 1995, 1998, 1999, 2000, 2001, siendo la mayor diferencia para el año 1998 con 1.11 partos (ee $=0.23 ; p=0.0001)$. Las vacas $C$ superaron de forma significativa $(p<0.05)$ a las ML en los años 1995, 2000 y 2001 con la mayor diferencia para el año 2000 con 1.15 partos (ee=0.31; $p=0.0002)$. Es importante destacar la clara disminución del número de partos a través de los años, con valores extremos entre el año 1995 y el 2006 para vacas CE de 4.13 partos $(e e=0.36 ; p<0.001)$.

\section{DISCUSIón}

Los factores que ejercieron influencia sobre la variación en VU y el promedio encontrado se encuentran en el rango inferior al reportado por diversos estudios realizados en Venezuela, donde obtuvieron un número de partos promedio entre 2.0 y 5.2 para vacas Bos taurus $x$ Bos indicus $(9,6-14)$. Sin embargo, todos estos estudios utilizaron como poblaciones de estudio las vacas que ya tenían al menos un parto, y por el contrario en la presente investigación se incorporó en la base de datos todas las hembras expuestas al programa de reproducción de cada finca.

Las haciendas bajo estudio ( $V V$ y $A G$ ) están ubicadas en una misma zona geográfica, donde las condiciones climáticas, recursos naturales 
y grupos raciales son similares, y un patrón de descarte similar lo que pudiera explicar por que no existió diferencias entre ellas. Este hallazgo, no coincide con lo reportado por Florio (9) quien expresa que para vida útil la variación más importante es la finca con diferencias extremas de hasta 2 a 5 partos, en un estudio realizado para 11 fincas ubicadas en zonas geográficas de Venezuela, mucho más contrastantes desde el punto de vista climático, así como también las diferencias tecnológicas entre las fincas estudiadas por Florio (9).

El efecto del A1P varía de un año a otro, debido a las condiciones ambientales (principalmente precipitación, temperatura y su relación con la humedad relativa), de manejo o las políticas de selección o descarte de las vacas. Tambien los planes sanitarios y económicos que pueden incidir en el tiempo, resultados que concuerdan con los obtenidos por Ponce de León y Guzmán (8), y Florio (9). En la tabla 3, se puede observar una tendencia decreciente de vida útil a lo largo de los años. Una situación particular con respecto a estos rebaños es que a partir del año 2005 en ambas haciendas se intensificó el descarte por bajo desempeño reproductivo y/o baja producción, lo que trajo como consecuencia una disminución en la oportunidad de las vacas de volver a parir y por ende una disminución en la VU.

Lo anterior, se puede observar también en el número de vacas que salieron del rebaño donde las que parieron en el 2005 y 2006 salieron más rápidamente del rebaño con 100 y 106 vacas (Tabla 1), respectivamente. Esto posiblemente este dado, por el hecho de que en el último trimestre del año 2003 se inició un programa descarte más riguroso por producción y reproducción. Por supuesto, esta tendencia también tiene que ver con el número de oportunidades que tuvieron las vacas, ya que las vacas que parieron por primera vez en el 2006 tuvieron tres oportunidades máximo de expresar su potencial en contra de vacas de años anteriores, puesto que en el presente trabajo se utilizó el concepto de grupo de oportunidad descrito por Hudson y Van Vleck (11) y fue limitado a tres oportunidades.

Las variaciones entre E1P pueden estar relacionadas con los cambios ambientales, de alimentación y de manejo que ocurren de una época a otra, lo que influye directamente en el número de partos estimados en la vida de las vacas. Los resultados coinciden con los reportados por Florio (9) quien no encontró efecto de época probablemente relacionado con el hecho de que en su estudio hubo haciendas ubicadas en zonas ecológicas contrastantes, mientras que el presente estudio sólo son dos haciendas con clima similar. En este caso las vacas que parieron de enero a marzo tuvieron un mayor número de partos, lo cual probablemente se debe a que, a pesar de ser una época de menor precipitación, le antecede una época de mayor disponibilidad de forraje, ya que las vacas que paren en esta época presentan mejor condición corporal al momento del parto, lo que les permite enfrentar mejor la lactancia y recuperarse más rápido para una posible gestación, mientras que el comportamiento de las vacas para la época septiembre-diciembre puede estar asociado a el estado de stress de la vaca ya que para estos meses cuando se registran las mayores precipitaciones, esto conlleva a un aumento en la proliferación de plagas, y mayor humedad relativa ambiental y mayor exceso de humedad en los suelos.

El resultado de GR coincide con lo expuesto por Florio (9) y Chirinos et al (15), quienes expresan que los animales con $50 \%$ de herencia europea superan en vida útil al Cebú. El número medio de partos obtenido para $C$ fue mayor a los reportados por Chirinos et al (15), resultando muy cercanas a las CE con las cuales no presentaron diferencias estadísticamente significativas. El hecho de que las vacas $C$, ocupen el segundo lugar en vida útil podría estar relacionado al hecho en que este grupo racial presenta una mejor reproducción como ha sido indicado por Pino et al (7), quienes afirman que vacas $C$ tuvieron intervalos entre partos 19 y 26 días menores que vacas $C E$ y $M L$, respectivamente, lo que posiblemente hace que el productor tienda a retener este tipo de animales en el sistema productivo a pesar de tener una menor producción de leche como fue también indicado por estos mismos autores. Vacas ML ocuparon el tercer lugar pudiendo indicar que cruces indiferenciados no favorecen la vida útil de las vacas.

Los resultados encontrados para la interacción $\mathrm{H}$ $x$ GR son similares a los expuestos por Florio (9), donde expone que los animales CE tuvieron en general mayor $\mathrm{VU}$, pero de acuerdo con ciertas diferencias en el manejo, criterios de selección del productor y condiciones climáticas puede producir cambios en las posiciones de los grupos raciales en las haciendas, de la misma manera puede incidir sobre el hecho de que un mismo grupo racial se puede comportar diferente de una hacienda a otra. Es probable que el manejo afecte de forma diferente. Un mejor manejo puede favorecer a vacas con mayor capacidad lechera en relación a las vacas CE. Probablemente el comportamiento tan contrastante de vacas ML entre fincas pueda deberse a los animales de 
fundación de cada rebaño en estudio (origen genético) presente en cada hacienda, lo que pudo provocar que a pesar de que ambos grupos sean $M L$, estos sean lo suficientemente diferentes desde el punto de vista de su constitución genética.

La variabilidad encontrada para la interacción GR x A1P pudiera estar relacionada con los efectos clima y políticas de descarte de las vacas ya que todos los grupos raciales siguen la misma tendencia, probablemente relacionada con el hecho de que el criterio más importante de descarte de hembras es la reproducción lo cual ejerce una presión importante en la salida de las vacas del rebaño. No se encontró en la literatura revisada trabajos que hayan considerado este efecto en la variación de la vida útil de vacas doble propósito.

En conclusión los valores promedio de VU es bajo, lo que podría comprometer tanto el avance genético como la rentabilidad del sistema productivo; sin embargo, se encuentran dentro del rango reportado en la literatura, a pesar de que en todas las investigaciones de referencia la población base de vacas fueron aquellas que al menos habían parido una vez, mientras que en la presente investigación la población base fue toda hembra que había sido incluida en el programa reproductivo.

La variación observada en VU de vacas doble propósito estuvo influenciada de forma significativa tanto por factores no genéticos como: el año y época de primer parto, como por factores genéticos como el grupo racial de la vaca. Sin embargo, la hacienda no afectó a la vida productiva. También, existió influencia significativa de las interacciones de hacienda por grupo racial y año de primer parto por grupo racial.

Las vacas con $50 \%$ de herencia europea y las vacas acebuadas superaron en vida útil a las vacas mestizas lecheras. Sin embargo, esta tendencia no fue similar para ambas fincas debido a la presencia de la interacción hacienda x grupo racial. De la misma manera, el comportamiento de estos grupos raciales tampoco fue similar para todos los años de estudio debido a la presencia de la interacción de año de primer parto $x$ grupo racial.

\section{REFERENCIAS}

1. Fundación para el Desarrollo de la Ganadería Bovina de Doble Propósito. Nuestra Ganadería de Doble Propósito. En: González Stagnaro, C., E Soto y L Ramírez Iglesia (Ed.). Avances en la Ganadería Doble Propósito. Maracaibo, Venezuela: Ediciones Astro Data S.A.; 2002.

2. Chirinos Z. Causas de eliminación y estrategias para mejorar la vida productiva del rebaño doble propósito. Alcances y perspectivas en la mejora genética de la ganadería doble propósito. En: Memorias XLIII Reunión del GIRARZ, Maracaibo, Venezuela; 2006.

3. Essl A. Longevity in dairy cattle breeding: a review. Livest Prod Sci 1998 57:79-89.

4. Chirinos Z. Longevidad en Ganadería Doble Propósito. Avances en la ganadería doble propósito. Maracaibo, Venezuela: Ediciones Astro Data S.A.; 2002.
5. Martínez G. Algunas alternativas para la evaluación genética de la longevidad de vacas en los sistemas de producción de carne. En: XX Cursillo sobre bovinos de carne. Universidad Central de Venezuela, Facultad de Ciencias Veterinarias. Maracay, Venezuela; 2005.

6. Murgueito E. Sistemas sostenibles de doble propósito como alternativa para la economía campesina. [fecha de acceso 02 de febrero de 2010] Livestock Research for Rural Development. 1992 4(3). URL disponible en: http://www.Irrd.org/lrrd4/3/enrique1.htm.

7. Pino T; Martínez G, Galíndez R, Castejón M, Tovar A. Efecto del grupo racial y algunos factores no genéticos sobre la producción de leche e intervalo entre partos en vacas de doble propósito. Rev Fac Cienc Vet 2009; 50(2):93-104.

8. Ponce de León R, Guzmán G. Heredabilidades y factores que afectan la longevidad y reproducción de por vida de vacas Holstein. Rev Cubana Cienc Agric 1991; 25:237-243. 
9. Florio J. Vida útil, permanencia y causas de salida en vacas doble propósito: su relación con factores no genéticos, grupo racial y valor genético estimado. [Trabajo de Maestría]. Maracay, Venezuela: Universidad Central de Venezuela; 2000.

10. Ewel J, Madriz A, Tosi JA. Zonas de vida de Venezuela. Memoria explicativa sobre el mapa ecológico. 2 ed. Caracas, Venezuela: Editorial Sucre; 1976.

11. Hudson GF, L. D. Van Vleck LD. Relationship between production and stayability in Holstein cattle. J Dairy Sci 1981; 64: 2246-2250.

12. Statistical Analysis System (SAS 9.1). [fecha de acceso 25 de junio de 2007].SAS Institute Inc., SAS 9.1, Cary, NC: 2004. URL disponible en: http://support.sas.com/ documentation /onlinedoc/91pdf/index.html
13. Snedecor GW, Cochran WG. Stadistical Methods. 8 ed. USA: Iowa State University Press. Ames; 1989.

14. Cardozo R, Vaccaro L. Vida útil en sistemas intensivos de producción de leche en el trópico. 2. Hembras mestizas europeas $x$ cebú. En: Memorias III Congreso Venezolano de Zootecnia. San Cristóbal, Venezuela; 1993.

15. Chirinos Z, C González- Stagnaro, MadridBury N, Rivera J. Vida útil, longevidad y causas de salida en vacas mestizas de doble propósito. Rev Científ FCV-LUZ 1999; IX(6): 477-484. 